\title{
The Genetics of Skin Color Variation in Farm Animals
}

\author{
Song TZ1 ${ }^{1}$, Chen $\mathrm{XY}^{1}$, Wang $\mathrm{GF}^{2}$, Zhou $\mathrm{P}^{2}$ and Ren $\mathrm{HX}^{2 *}$ \\ ${ }^{1}$ Institute of animal science, Tibet Academy of Agricultural and Animal Husbandry \\ Sciences, China \\ ${ }^{2}$ Chongqing Academy of Animal Sciences, China
}

Review Article

Volume 2 Issue 3

Received Date: May 15, 2019

Published Date: May 22, 2019

DOI: $10.23880 /$ izab-16000151

*Corresponding author: Hang Xing REN, Chongqing Academy of Animal Sciences, Chongqing, Rongchang 402460, China, Tel: +86 023-46793019; Email: rhxe@163.com

\begin{abstract}
Melanin from melanocytes has a broad spectrum of biological activities, including protection against enzymatic lysis, UV radiation, and damage by oxidants and resistance to drugs by pathogens, protection of insects against bacteria and antiviral protection, etc. Coloration of the hair, skin, and eye in animal depends on the quantity, quality, and distribution of the pigment in tissues. Pigmentation is a variety of phenotypes that is important to husbandry. We reviewed the progress of skin color variation in farm animals to provide insights into the biology of skin pigmentation and melanocyte.
\end{abstract}

Keywords: Melanin; Skin; Pigmentation; Candidate Gene; Farm Animal

\section{Pigment and its Potential Function}

\section{Melanogenesis}

Coloration of the hair, skin, and eye in animal depends on the quantity, quality, and distribution of the pigment in tissues. Melanocytes are pigment producing cells of the skin in humans and other vertebrates. Melanocytes originate from the neural crest with pluripotential cells that gradually become lineage specific during development, eventually they become localized in hair follicles as well as in the epidermis to pigment the hair and skin, respectively [1-3]. It is known that melanocyte is not only responsible for synthesis of different types of pigment in melanosomes, but also for the transport of pigment from melanocyte to the surrounding epithelial cell (keratinocyte). Melanins can be produced in two chemically distinct types, black-to-brown eumelanin and yellow-to-reddish-brown pheomelanin by the melanocyte in mammal and bird. The eumelanins are highly insoluble pigments that form within specialized cells known as melanocytes. Enzymatic action of the enzyme tyrosinase on the amino acid tyrosine produces melanin. In their primary biosynthetic pathway, tyrosine is hydroxylated to form the catecholamine 3,4-dihydroxyphenylalanine (DOPA), which is then oxidized to form 3,4dioxyphenylalanine (dopaquinone) before cyclization to 5,6-indole quinones and their subsequent polymerization to form melanin. Similar to the biosynthesis of eumelanin, melanin known as pheomelanin is biologically synthesized, except that a precursor containing Sulphur is incorporated in the structure. In nature, many biological systems produce a combination of the two types of melanin. In husbandry, pigmentation phenotypes are a kind of important production traits, especially as a distinct marker for certain breed of animals.

\section{Potential Functions of Melanin}

It has been reported that melanin from natural sources has a broad spectrum of biological activities, including protection against enzymatic lysis, UV radiation, damage by oxidants, resistance to drugs by pathogens, protection of insects against bacteria and antiviral 


\section{International Journal of Zoology and Animal Biology}

protection [3-6]. Melanin also can chelate metal ions and to act as a physiological redox buffer $[7,8]$. Evidence from Revskaya, et al. [4] and Kunwar A, et al. [5] indicate that melanin from the plant and fungus can provide significant protection against radiation in mice. In addition, melanin can interact with drugs and metals and has certain pharmacological properties. The most significant properties of melanin is its antioxidant effects and enhancement and modulation of the immune system. Recently, other important and valuable characteristics of melanin have been identified, such as modulation of gastrointestinal health, hepatoprotective effects, antiinflammatory effects, and anti-carcinogenic effects [6].

\section{Candidate Genes for Skin Color in Farm Animals}

\section{Chicken}

The Silky chicken (Gallus gallus) is the earliest studied and noteworthy for the hyperpigmentation in tissues and organs such as the dermal layer of skin, bone, muscle, pleura, trachea, blood vessels, abdominal lining, and connective tissue. Silky is an exceptional chicken in which numerous melanoblasts travel via a ventral pathway and disperse into internal organs. Finally, these ectopic melanocytes induce heavy dermal and visceral melanization known as Fibromelanosis (Fm). Identification of the candidate gene for Fm locus continued over half of a century, until the breakthrough undertaken by Dorshorst, et al. [7] It has been demonstrated that the causal mutation of $\mathrm{Fm}$ is an inverted duplication endothelin 3 (EDN3) gene in genomic regions, which increase expression in EDN3, thus promoting melanoblasts migration and proliferation in early embryonic stage [7]. Interesting, Yu, et al. [8] have identified a novel mutation (c.-1826A $>$ T), associated with the skin color (dorsal and subalar) of black-bone chicken, in the ASIP gene promoter by altering ASIP transcriptional activity [8]. This indicates ASIP participates in the regulation of skin color, which is supported by the recent study in zebrafish and avain $[9,10]$. Li, et al. [11] used the $600 \mathrm{~K}$ Affymetrix Axiom HD genotyping array to perform a genome-wide association study on pure lines of 19 Tibetan hens with dermal pigmentation shank and 21 Tibetan hens with yellow shank to refine the Id location. The genome-wide study revealed that 3 SNP located at 78.5 to $79.2 \mathrm{Mb}$ on the Z chromosome in the current assembly of chicken genome (galGal4) were significantly associated with dermal shank pigmentation of chickens, but none of them were located in known genes. The interval we refined was partly converged with previous results, suggesting that the Id gene is in or near our refined genome region [11]. Zhang, et al. [12] investigated the genetic basis of the gray dilution phenotype in the Anti tile-like gray chicken. They found that the allele E of the MC1R gene and FM alleles act together to cause the development of the "five-black" phenotype (black feather, comb, skin, shank, and beak), whereas the MLPH mutation results in defective melanosome transport, leading to the development of the "five-gray" phenotype [12]. In additional, Zhang, et al. [13] mapped the gene responsible for the dermal shank pigmentation in chickens by an association analysis and a differential expression analysis, and found that GRAMD3 could be the most likely candidate gene for the Id locus.

\section{Pigs}

The belt pattern can be described as a white band of varying width around the midsection of the body but does not always encircle the body completely, and is considered to result from a lack of melanocytes. Giuffra, et al. [14] confirmed the dominant inheritance of the belt pattern in Hampshire swine and identified the belt locus as the fourth allele at the KIT locus on pig chromosome 8. Fernandez, et al. [15] reported that two OCA2 intragenic haplotypes were associated with skin color variation in Iberian pigs, which provide evidence of a suggestive dominant effect of haplotypes on color intensity and indicate an important contribution of additive polygenic effects (h2 $=0.56+/-0.21)$ to the variance of this trait. The phenotype of ACOP (ambilateral circumocular pigmentation) is characteristic for some breeds of farm animal, such as Rongchang pig and a minority of the FV animals of the Fleckvieh (FV) cattle breed. In areas where animals are exposed to increased solar ultraviolet radiation, ACOP is associated with a reduced susceptibility to bovine ocular squamous cell carcinoma (BOSCC, eye cancer). Interestingly, Chen, et al. [16] found a short insertion in the distal melanocyte-specific regulatory region of MITF creates a de novo silencer that completely eliminated the expression of the transcripts for the MITF-M isoform, which led to observed phenotypes of deafness and skin depigmentation, similar to the phenotype of Waardenburg syndrome in humans. $\mathrm{Xu}$, et al. [17] compared expression profiles of coding and non-coding RNAs from white and black skin in Wuzhishan pigs using high-throughput RNA sequencing method. They demonstrated that key genes such as MLANA, PMEL, TYR, TYRP1, DTC, TRPM1 and CAMK2A had significantly different levels of expression in the two skin tissues, and that a total of 15 lncRNAs, 11 miRNAs and 7 genes formed 23 IncRNA-miRNA-gene pairs, suggesting that complex regulatory networks of coding and non-coding genes underlie the coat color trait in Wuzhishan pigs. 


\section{International Journal of Zoology and Animal Biology}

\section{Cattles}

Pausch, et al. [18] identified QTL point to MCM6, PAX3, ERBB3, KITLG, LEF1, DKK2, KIT, CRIM1, ATRN, GSDMC, MITF and NBEAL2 as underlying genes for eye area pigmentation in cattle. The twelve QTL regions explain $44.96 \%$ of the phenotypic variance of the proportion of daughters with ACOP. The chromosomes harboring significantly associated SNPs account for $54.13 \%$ of the phenotypic variance, while another $19.51 \%$ of the phenotypic variance is attributable to chromosomes without identified QTL. These support a polygenic inheritance pattern of ACOP in cattle and provide the basis for efficient genomic selection of animals that are less susceptible to serious eye diseases. Color sidedness is a dominantly inherited phenotype of cattle characterized by the polarization of pigmented sectors on the flanks, snout and ear tips. It is also referred to as 'lineback' or 'witrik' (which means white back), as color-sided animals typically display a white band along their spine. Color sidedness is documented at least since the Middle Ages and is presently segregating in several cattle breeds around the globe, including in Belgian blue and brown Swiss. Durkin, et al. [19] reported a novel CNV-generating translocation mechanism involving circular intermediates, namely, color sidedness is determined by a first allele on chromosome 29 (Cs(29)), which results from the translocation of a 492-kilobase chromosome 6 segment encompassing KIT to chromosome 29, and a second allele on chromosome $6(\operatorname{Cs}(6))$, derived from the first by repatriation of fused 575-kilobase chromosome 6 and 29 sequences to the KIT locus. Awasthi Mishra, et al. [20] and Rothammer, et al. [21] identified TWIST2 as the candidate gene of the belted phenotype in Brown Swiss. Hofstetter, et al. [22] found a non-coding regulatory variant in the 50region of the MITF gene is associated with white-spotted coat in Brown Swiss cattle. Zwane, et al. [23] reported that KIT and MITF were associated with skin pigmentation in three South African indigenous breeds (Afrikaner, Drakensberger, and Nguni) using whole genome sequencing.

\section{Sheep and Goats}

Penagaricano, et al. [24] identified candidate genes associated with the development of black skin spots in Corriedale sheep, and found that C-FOS, KLF4 and UFC1 could be candidate genes associated with the development of black skin spots. Raadsma, et al. [25] identified QTL of 13 skin and fibre pigmentation traits in sheep. A total of 19 highly significant, 10 significant and seven suggestive QTL were identified in a QTL mapping experiment using an Awassi $\times$ Merino $\times$ Merino backcross sheep population. They revealed that the ovine TYRP1 gene on OAR 2 was a strong positional candidate gene. Up to $47 \%$ of the observed variation in pigmentation was accounted for by models using TYRP1 haplotypes and 83\% for models with interactions between two QTL probabilities, offering scope for marker-assisted selection for these traits. The Youzhou dark goat is a natural mutant with dark skin over the whole body including the visible mucous membranes in China. We investigated the genetic basis of the skin hyperpigmentation in Youzhou dark goat [26-28]. Our findings suggest that a presumed structure variation (duplication or insertion) in ASIP might be responsible for its lower expression in the hyper pigmented skin (Youzhou dark goat) by determining the distribution of melanocytes across the body at early development stage, suggesting ASIP might be the key candidate gene for the skin hyperpigmentation in You zhou dark goat. In addition, two another interesting pigmentation phenotypes in sheep (Ovis aries) and goat(Capra hircus) have been reported recently, the black-boned sheep and goat, which characterized in hyperpigmentation of the muscle, bone surface (periosteum), kidney, inner skin, heart, lung and trachea, compared with the red coloration in normal animal [29,30]. Deng, et al. [29,31-33] and Jiang, et al. [34-36] investigated that polymorphism of the pigmentation genes and histological characteristics respectively, but the causative genes associated with the hyperpigmentation phenotype in Black-bone sheep and goat remain to be identified further.

\section{Conclusion and Perspective}

Unlike the model animal such as mice and rat, there are few melanocyte lines available for further investigations in farm animals, which hinder the further investigation of pigmentation in farm animals. In addition, most investigations of pigmentation phenotypes focus on variation of coat color rather than skin color in the livestock. However, investigations of the skin pigmentation can provide valuable information for human diseases associated with melanin, such as skin melanopathy, melanosis coli, mucosal melanosis, etc. The findings in mice and human may contribute much to our understanding of the genetic basis for skin color variation in farm animals, despite the fact that there are many differences in dermal microstructure between mice and human [37]. Especially, many important and valuable findings from mice in vivo and in vitro are milestones in this topic [38-49]. With the emerge of the state of the art in life science technology such as high throughput sequencing (PacBio and Manopore) and genome edition, it is more and more feasible for people to reveal the 


\section{International Journal of Zoology and Animal Biology}

molecular mechanisms underlying the phenotypes of skin color in farm animals.

\section{Acknowledgement}

This work was supported by the National Natural Science Foundation of China (No. 31672393), the Major Special Projects of Science and Technology Agriculture in Tibet Autonomous Region（XZ201801NB42）

\section{References}

1. Erickson CA, Reedy MV (1998) Neural crest development: the interplay between morphogenesis and cell differentiation. Curr Top Dev Biol 40: 177209.

2. Dorsky RI, Moon RT, Raible DW (1998) Control of neural crest cell fate by the Wnt signalling pathway. Nature 396(6709): 370-373.

3. Sommer L (2005) Checkpoints of melanocyte stem cell development. Science's signaling 2005(298): pe42.

4. Revskaya E, Chu P, Howell RC, Schweitzer AD, Bryan RA, et al. (2012) Compton scattering by internal shields based on melanin-containing mushrooms provides protection of gastrointestinal tract from ionizing radiation. Cancer biother radiopharm 27(9): 570-576.

5. Kunwar A, Adhikary B, Jayakumar S, Barik A, Chattopadhyay S, et al. (2012) Melanin, a promising radioprotector: mechanisms of actions in a mice model. Toxicol appl pharmacol 264(2): 202-211.

6. ElObeid AS, Kamal-Eldin A, Abdelhalim MAK, Haseeb AM (2017) Pharmacological Properties of Melanin and its Function in Health. Basic clin pharmacol toxicol 120(6): 515-522.

7. Dorshorst B, Molin AM, Rubin CJ, Johansson AM, Stromstedt L, et al. (2011) A complex genomic rearrangement involving the endothelin 3 locus causes dermal hyperpigmentation in the chicken. PLoS Genet 7(12): e1002412.

8. Yu S, Wang G, Liao J (2019) Association of a novel SNP in the ASIP gene with skin color in black-bone chicken. Anim Genet 50(3): 283-286.

9. Cal L, Suarez Bregua P, Comesana P, Owen J, Braasch I, et al. (2019) Countershading in zebrafish results from an Asip1 controlled dorsoventral gradient of pigment cell differentiation. Scientific reports 9(1): 3449.

10. Inaba $\mathrm{M}$, Jiang $\mathrm{TX}$, Liang $\mathrm{YC}$, Tsai $\mathrm{S}$, Lai $\mathrm{YC}$, et al. (2019) Instructive role of melanocytes during pigment pattern formation of the avian skin. PNAS 116(14): 6884-6890.

11. Li G, Li D, Yang N, Qu L, Hou Z, et al. (2014) A genomewide association study identifies novel single nucleotide polymorphisms associated with dermal shank pigmentation in chickens. Poult Sci 93(12): 2983-2987.

12. Xu JG, Xie MG, Zou SY, Liu XF, Li XH, et al. (2016) Interactions of allele $\mathrm{E}$ of the MC1R gene with FM and mutations in the MLPH gene cause the five-gray phenotype in the Anyi tile-like gray chicken. Genet mol res 15(2).

13. Xu J, Lin S, Gao X, Nie Q, Luo Q, et al. (2017) Mapping of Id locus for dermal shank melanin in a Chinese indigenous chicken breed. J genet 96(6): 977-983.

14. Giuffra E, Evans G, Tornsten A, Wales R, Day A, et al. (1999) The Belt mutation in pigs is an allele at the Dominant white (I/KIT) locus. Mamm Genome 10(12): 1132-1136.

15. Fernandez A, Silio L, Rodriguez C, Ovilo C (2006) Characterization of OCA2 cDNA in different porcine breeds and analysis of its potential effect on skin pigmentation in a red Iberian strain. Anim Genet 37(2): 166-170.

16. Chen L, Guo W, Ren L, Yang M, Zhao Y, et al. (2016) A de novo silencer causes elimination of MITF-M expression and profound hearing loss in pigs. BMC biology 14: 52 .

17. Xu Q, Liu X, Chao Z, Wang K, Wang J, et al. (2019) Transcriptomic Analysis of Coding Genes and NonCoding RNAs Reveals Complex Regulatory Networks Underlying the Black Back and White Belly Coat Phenotype in Chinese Wuzhishan Pigs. Genes (Basel) $10(3)$.

18. Pausch H, Wang X, Jung S, Krogmeier D, Edel C, et al. ( 2012) Identification of QTL for UV-protective eye area pigmentation in cattle by progeny phenotyping and genome-wide association analysis. PLoS One 7(5): e36346. 


\section{International Journal of Zoology and Animal Biology}

19. Durkin K, Coppieters W, Drogemuller C, Ahariz N, Cambisano N, et al. (2012) Serial translocation by means of circular intermediates underlies colour sidedness in cattle. Nature 482(7383): 81-84.

20. Awasthi Mishra N, Drogemuller C, Jagannathan V, Keller I, Wuthrich D, et al. (2017) A structural variant in the 5'-flanking region of the TWIST2 gene affects melanocyte development in belted cattle. PLoS One 12(6): e0180170.

21. Rothammer S, Kunz E, Krebs S, Bitzer F, Hauser A, Zinovieva N, et al. (2018) Remapping of the belted phenotype in cattle on BTA3 identifies a multiplication event as the candidate causal mutation. Genet Sel Evol 50(1): 36.

22. Hofstetter S, Seefried F, Hafliger IM, Jagannathan V, Leeb T, et al. (2019): A non-coding regulatory variant in the 5'-region of the MITF gene is associated with white-spotted coat in Brown Swiss cattle. Anim Genet 50(1): 27-32.

23. Zwane AA, Schnabel RD, Hoff J, Choudhury A, Makgahlela ML, et al. (2019) Genome-Wide SNP Discovery in Indigenous Cattle Breeds of South Africa. Frontiers in genetics 10: 273.

24. Penagaricano F, Zorrilla $\mathrm{P}$, Naya H, Robello C, Urioste JI (2012) Gene expression analysis identifies new candidate genes associated with the development of black skin spots in Corriedale sheep. J appl genet 53(1): 99-106.

25. Raadsma HW, Jonas E, Fleet MR, Fullard K, Gongora J, et al. (2013) QTL and association analysis for skin and fibre pigmentation in sheep provides evidence of a major causative mutation and epistatic effects. Anim Genet 44(5): 547-559.

26. Ren H, Wang G, Jiang J, Li J, Fu L, et al. (2017) Comparative transcriptome and histological analyses provide insights into the prenatal skin pigmentation in goat (Capra hircus). Physiol genom 49(12): 703711.

27. Ren H, Wang G, Chen L, Jiang J, Liu L, et al. (2016) Genome-wide analysis of long non-coding RNAs at early stage of skin pigmentation in goats (Capra hircus). BMC Genomics 17(1): 67.

28. Ren H, Wang G, Lu J, Li J, Jiang J, et al. (2015) The characteristic histomorphology of various skin colors and expression of genes involved in melanogenesis in goats. Acta Veterinaria et Zootechnica Sinica 46(9): 1525-1531.

29. Deng WD, Yang SL, Huo YQ, Gou X, Shi XW, et al. (2006) Physiological and genetic characteristics of black-boned sheep (Ovis aries). Anim Genet 37(6): 586-588.

30. Liu S, Liu G, Ruan X, Hu J, Huang C, et al. (2010) Study on the new characteristics of the black-bone goat China Herbivore 2010: 375-377.

31. Deng WD, Shu W, Yang SL, Shi XW, Mao HM (2009) Pigmentation in Black-boned sheep (Ovis aries): association with polymorphism of the MC1R gene. Mol Biol Rep 36(3): 431-436.

32. Deng W, Tan Y, Wang X, Xi D, He Y, et al. (2009) Molecular cloning, sequence characteristics, and polymorphism analyses of the tyrosinase-related protein 2/DOPAchrome tautomerase gene of blackboned sheep (Ovis aries). Genome 52(12): 10011011.

33. Deng WD, Xi DM, Gou X, Yang SL, Shi XW, et al. (2008) Pigmentation in Black-boned sheep (Ovis aries): association with polymorphism of the Tyrosinase gene. Mol Biol Rep 35(3): 379-385.

34. Han YG, Liu GQ, Jiang XP, Liang GM, He CB, et al. (2013) Investigation of individual heterozygosity correlated to growth traits in Tongshan Black-boned goat. Mol Biol Rep 40(11): 6075-6079.

35. Xu F, Jiang X, Liu G, Xie G, Zhang T, et al. (2015) The origin of melanin in muscular tissue of black-boned goat. Chinese Journal of Animal Science 51(11): 1013.

36. Xie G, Liu G, Jiang X, Hu X, Wang J (2014) Location and characteristics of melanocytes of black-boned goat skin. Journal of Huazhong Agricultural University 33(2): 83-88.

37. Nishimura EK (2011) Melanocyte stem cells: a melanocyte reservoir in hair follicles for hair and skin pigmentation. Pigment cell \& melanoma res 24(3): 401-410.

38. Van Raamsdonk CD, Fitch KR, Fuchs $H$, de Angelis $M H$, Barsh GS (2004) Effects of G-protein mutations on skin color. Nat genet 36(9): 961-968. 


\section{International Journal of Zoology and Animal Biology}

39. Bennett DC, Lamoreux ML (2003) The color loci of mice-a genetic century. Pigment Cell Res 16(4): 333344.

40. Fitch KR, McGowan KA, van Raamsdonk CD, Fuchs H, Lee D, et al. (2003) Genetics of dark skin in mice. Genes dev 17(2): 214-228.

41. Mallarino R, Henegar C, Mirasierra M, Manceau M, Schradin C, et al. (2016) Developmental mechanisms of stripe patterns in rodents. Nature 539(7630): 518523.

42. Garcia RJ, Ittah A, Mirabal S, Figueroa J, Lopez L, et al. (2008) Endothelin 3 induces skin pigmentation in a keratin-driven inducible mouse model. J Invest Dermatol 128(1): 131-142.

43. McGowan KA, Li JZ, Park CY, Beaudry V, Tabor HK, et al. (2008) Ribosomal mutations cause p53-mediated dark skin and pleiotropic effects. Nat genet 40(8): 963-970.

44. Van Raamsdonk CD, Barsh GS, Wakamatsu K, Ito S (2009) Independent regulation of hair and skin color by two G protein-coupled pathways. Pigment Cell \& Melanoma Res 22(6): 819-826.
45. Aoki H, Yamada Y, Hara A, Kunisada T (2009) Two distinct types of mouse melanocyte: differential signaling requirement for the maintenance of noncutaneous and dermal versus epidermal melanocytes. Development 136(15): 2511-2521.

46. Hou L, Panthier JJ, Arnheiter H (2000) Signaling and transcriptional regulation in the neural crest-derived melanocyte lineage: interactions between KIT and MITF. Development 127(24): 5379-5389.

47. Lin CB, Babiarz L, Liebel F, Roydon, Price E, et al. (2002) Modulation of microphthalmia-associated transcription factor gene expression alters skin pigmentation. J Invest Dermatol 119(6): 1330-1340.

48. Weiner L, Han R, Scicchitano BM, Li J, Hasegawa K, et al. (2007) Dedicated epithelial recipient cells determine pigmentation patterns. Cell 130(5): 932942.

49. Takeo M, Lee W, Rabbani P, Sun Q, Hu H, et al. (2016) EdnrB Governs Regenerative Response of Melanocyte Stem Cells by Crosstalk with Wnt Signaling. Cell reports 15(6): 1291-1302. 